\title{
Correction to: Efects of high-flow oxygen therapy on patients with hypoxemia after extubation and predictors of reintubation: a retrospective study based on the MIMIC-IV database
}

Taotao Liu', Qinyu Zhao ${ }^{2}$ and Bin Du 3* $^{*}$

\section{Correction to: BMC Pulm Med(2021) 21:160}

https://doi.org/10.1186/s12890-021-01526-2

Following publication of the article, the authors flagged that the article had published with incorrect affiliation details in the author list.

The article has since been corrected and the updated author list can be found in this correction.

\section{Author details}

'Department of Surgical Intensive Care Unit, Beijing Hospital, National Center of Gerontology, Institute of Geriatric Medicine, Chinese Academy of Medical Sciences, Beijing 100730, China. ${ }^{2}$ College of Engineering and Computer Science, Australian National University, Canberra 2600, Australia. ${ }^{3}$ Department of Medical Intensive Care Unit, State Key Laboratory of Complex Severe and Rare Diseases, Peking Union Medical College Hospital, Chinese Academy of Medical Science and Peking Union Medical College, 1 Shuai Fu Yuan, Beijing 100730, China.
Published online: 09 June 2021

\section{Reference}

1. Liu T, Zhao Q, Du B. Effects of high-flow oxygen therapy on patients with hypoxemia after extubation and predictors of reintubation: a retrospective study based on the MIMIC-IV database. BMC Pulm Med. 2021;21:160. https://doi.org/10.1186/s12890-021-01526-2.

\section{Publisher's Note}

Springer Nature remains neutral with regard to jurisdictional claims in published maps and institutional affiliations. 